\title{
La reprise du discours des autres dans les interactions pédagogiques plurilingues en ligne : une affaire de genre(s) ?
}

\author{
Devilla, Lorenzo \\ Université de Sassari (Italie) \\ Laboratoire LIDILEM (Université Stendhal-Grenoble3) \\ ldevilla@uniss.it
}

\section{Introduction}

L'interaction verbale dans les genres électroniques est de nature particulière. Dans les forums de discussion, qui feront l'objet de la présente étude, deux ou plusieurs interlocuteurs interagissent par le biais des messages envoyés (Mangenot, 2007). En particulier, l'interaction est différée dans le temps, la seconde partie d'une paire adjacente (du type question-réponse) pouvant être produite longtemps après l'intervention initiative, c'est pourquoi on parle aussi d'échanges asynchrones. Même si de nombreux messages asynchrones ont une forte dimension monologale, dans la mesure où ils ne paraissent pas susciter l'enchaînement, la citation d'extraits de discours d'un autre participant (ou de plusieurs autres) est une pratique récurrente dans les discussions en ligne (Lucas, 2010 ; Marcoccia, 2010). La reprise du discours de l'autre y introduit une dimension séquentielle et interactionnelle (Mondada, 1999). Elle contribue à recréer les conditions de la conversation réelle (von Münchow 2004 ; von Münchow et Rakotoelina, 2006), limitant la discontinuité thématique de l'échange dialogique (Hert 1999) - ou, comme le soulignent certains chercheurs, polylogique (Marcoccia, 2004) -, compensant l'absence d'interactivité directe (Herring, 2001 ; Marcoccia, 2010). Les participants à ces échanges invoquent aussi des autorités discursives autres. Les citations d'autorité, dont les sources sont les plus variées, sont fréquentes dans leurs messages.

Dans cet article, nous nous proposons donc d'analyser ces formes de citations dans un corpus d'interactions pédagogiques liées au projet Galanet (www.galanet.eu), dont le but est de favoriser l'intercompréhension en langues romanes. Y aurait-il des conduites langagières influencées par le «genre » (au sens d'identité sociale de sexe, «gender» en anglais)? S'il est vrai, comme l'affirment Holmes et Schnurr (2006 : 26), que le discours est imbibé des différences de genre (« our discourse is drenched in gender »), les forums de discussion pédagogiques font-ils émerger différentes façons de rapporter les discours en rapport avec le paramètre du sexe ? En effet, plusieurs études ont déjà relevé dans la conversation électronique les mêmes différences de "genre » que dans les discours parlés, notamment en ce qui concerne l'expression des émotions et de la politesse (voir Herring, 2000). Il s'agira donc ici d'interroger notre corpus sur ces aspects.

\section{Les études sur le " genre »}

Depuis les années 70, notamment à la suite des travaux fondamentaux de Robin Lakoff (1973) et de Mary Richie Key (1974), beaucoup de chercheurs dans le domaine des «Gender and language studies » ont analysé le rapport entre les rôles sexuels et la langue. On a ainsi mis en évidence qu'il existe un langage des femmes avec des caractéristiques bien précises que l'on peut répertorier: l'utilisation d'une intonation interrogative posée à la fin d'une assertion, les stratégies de mitigation d'une déclaration (Well, I don't know...), la présence d'intensificateurs (so) et de marques de politesse dans la conversation, l'absence d'humour, la présence l'hypercorrection, etc. (Greco, 2011). Dans son ouvrage pionnier, Lakoff a observé aussi que les femmes tendent à utiliser des formes standard plus fréquemment que les hommes. Cet aspect émerge également des travaux en sociolinguistique de Trudgill (1972) sur la variation phonologique dans le parler de Norwich. Les formes du standard sont employées plus par les femmes que 
par les hommes, ceux-ci s'identifiant davantage avec les formes populaires et stigmatisées. Pour Trudgill, le choix de la variété de prestige serait dû à la position d'infériorité de la femme dans la société (cf. Eckert 1989 : 249). Le paradigme du «déficit» est invoqué également par Lakoff pour expliquer les caractéristiques du parler des femmes. Or, l'approche de Trudgill a été critiquée notamment par Milroy (1980) et Eckert (2000) pour qui le paramètre « gender » entre en relation respectivement avec les réseaux sociaux (denses vs lâches) et la classe sociale. De plus, les locuteurs choisissent certaines façons de parler comme un "acte d'identité » afin de ressembler au groupe auquel ils souhaitent s'identifier. Dans ses études des conversations de «teenagers » réalisées à Londres, Jennifer Coates (1994), précurseuse des travaux sur la présentation de soi (voir Baider, 2012) remarque des patrons de changement dans les styles discursifs tout au long de l'année scolaire. L'innovation et l'agencité («agency» en anglais) dans le discours sont ici interprétées en termes d'entrée des filles dans l'adolescence.

Dans la littérature sur les échanges discursifs, on découvre également qu'il existe des styles interactionnels transmis dès l'enfance lors des procédés de socialisation langagière (Greco, 2011). Ainsi, les hommes auraient-ils tendance à mettre en avant dans la conversation un style marqué par la compétition et par l'opposition, alors que celui utilisé par les femmes relèverait davantage de l'intimité et du partage (Tannen, 1991, cité in Greco, 2011). Les femmes auraient aussi tendance à coopérer avec leurs interlocuteurs (Holmes 1989), elles auraient davantage recours à la politesse positive (Holmes 1995) et emploieraient un style indirect dans l'interaction (Tannen, 1995). Toutefois, cette approche essentialiste et binaire des sexes et des genres a été dépassée par les études post-modernes et les études queer (queer linguistics) à partir des années quatre-vingt-dix, dans le sillage des travaux de Judith Butler (1990) (cf. Baider, 2012; Greco, 2011). Cette approche théorique, qui domine les travaux actuels anglo-saxons, focalise davantage sur la façon dont les normes liées au genre sont construites ou déconstruites dans le discours que sur les différences basées sur le genre ${ }^{1}$. L'accent est mis sur le contexte, qui peut créer, effacer ou renverser les différences relevant du genre (Bell et alii, 2006). Ainsi, a-t-on remarqué, par exemple, que les interruptions sont plus nombreuses dans le cadre d'une conversation entre amis que dans le cadre d'une conversation avec des étrangers (Ibid.). L'analyse d'un corpus de 54 textes répondant à la question « Can this marriage be saved? » sur le site Ladies home journal a montré que dans le cadre d'un conflit conjugal il n'y a pas de différences de genre quant à l'emploi de mots exprimant une émotion positive ou négative. Cela confirme « that context does play an important role in predicting which gender will use a particular language strategy» (Ibid.). De même, les femmes peuvent avoir recours à un discours et à des stratégies discursives normalement associées aux hommes. C'est le cas des joueuses de tennis adoptant le modèle conversationnel agressif normalement attribué aux hommes (Sznycer, 2010). Il est vrai aussi l'inverse : les hommes peuvent employer des stratégies de politesse normalement associées aux femmes, à l'instar de Sarkozy lors du débat de la présidentielle de 2007 (Fracchiolla, 2011). En l'occurrence, comme le montre Fracchiolla, l'excès de politesse de la part du candidat Sarkozy est une stratégie sciemment utilisée pour mettre l'accent sur la différence des sexes et sur l'incapacité de la candidate Royal à occuper un rôle si important.

\section{Genre, discours et communication électronique}

Or, qu'en est-il dans la communication électronique, qui nous intéresse de près ici? Dans la communication médiatisée par ordinateur ( $\mathrm{CMO}, \mathrm{CMC}$ en anglais), les phénomènes relevant du genre sont ceux que l'on a déjà observés dans la communication en face-à-face (Herring, 2000). Par exemple, dans la communication asynchrone, dont relèvent les forums de discussion, il est plus probable que les hommes postent des messages plus long. De plus, ce sont eux qui normalement ouvrent et ferment les fils de discussion dans des groupes mixtes (hommes-femmes). Ils affirment leurs propos de manière ferme et décidée. En général, dans l'interaction, les hommes sont plus enclins au conflit : «in general, [they] manifest an adversarial orientation towards their interlocutors » (Herring, 2000). En revanche, les femmes ont tendance à écrire des messages plus courts, à étayer et justifier leurs affirmations ainsi qu'à s'excuser. Elles adoptent également une attitude collaborative envers leurs interlocuteurs : «in general, [they] manifest an 'aligned' orientation towards their interlocutors » (Ibid.). Herring tient pourtant à souligner que cela ne veut pas dire que chaque homme ou chaque femme manifeste toujours ces comportements 
dans ses pratiques discursives. D'ailleurs, des exceptions peuvent être repérées. Toujours est-il que le genre permet de prédire certaines pratiques langagières avec plus de précision : «It does mean, however, that gender predicts certain online behaviors with greater than chance frequency when considered over aggregate populations of users » (Herring, 2000). Dans le domaine de la CMO, les blogs méritent des considérations particulières, compte-tenu de la nature particulière de ce genre électronique, sorte de journal personnel en ligne. Les travaux d'Herring et Paolillo (2006) sur la fréquence de certains items lexicaux ont en effet montré que de ce point de vue il n'y a pas de différenciation selon le paramètre " gender ». Cependant, si l'on prend en considération les commentaires postés par les internautes directement sous la note publiée dans le blog, c'est-à-dire lorsque le blog est étudié en tant qu'espace d'interaction entre le blogueur et les lecteurs, on retrouve dans les blogs aussi les différences discursives et interactionnelles relevées dans la CMO (Herring et Paolillo, 2006). D'autre part, une étude menée sur des blogs d'adolescents (Huffaker et Calvert, 2005) a pourtant montré qu'il n'y a pas de différences entre les blogs créés par des hommes et ceux créés par des femmes. L'aisance dans l'usage de cet outil de communication expliquerait le nivellement des différences de genre. Les auteurs de cette recherche avancent également que la nouvelle génération d'internautes est peut-être en train de devenir plus «androgyne » dans ses pratiques communicatives en ligne. Cela serait confirmé par le fait que les adolescents, contrairement à ce qui a été remarqué dans les études précédentes sur les chats et les forums, utilisent en moyenne plus d'émoticônes que les adolescentes. De même, les adolescentes n'emploient pas un langage plus accommodant et collaboratif, tel qu'il a été en revanche décrit dans les travaux précédents portant sur les caractéristiques du langage des femmes (Eckert et McConnell-Ginet, 2003; Herring, 2000; Savicki et alii, 1996).

\section{Dispositif de recueil de données et choix du corpus}

\subsection{La plateforme Galanet}

Le corpus d'interactions pédagogiques qui feront l'objet de cette étude est tiré de la plateforme Galanet, dont le but est de favoriser l'intercompréhension en langues romanes (français, espagnol, italien, portugais et roumain) $)^{2}$. Par intercompréhension on entend la possibilité pour un locuteur de communiquer avec des interlocuteurs alloglottes en utilisant sa langue (romane) de référence et en développant sa capacité à comprendre les langues (romanes) de ses interlocuteurs. Le scénario d'apprentissage des formations Galanet a été pensé en référence au principe didactique actionnel, conformément aux préconisations du Cadre commun européen de référence pour les langues (2001 : 15). L'accent est mis sur la co-construction par étapes successives d'une tâche collective, qui consiste à réaliser et à publier un « dossier de presse » plurilingue sur le net. L'action commune est censée stimuler l'interaction entre les apprenants, qui à son tour devrait favoriser le développement des compétences réceptives et interactives. Plus précisément, le scénario d'apprentissage se déroule en quatre phases distinctes, chacune se donnant un objectif opérationnel clairement défini (Masperi et Quintin, 2007) :

Tableau 1 - Le scénario d'apprentissage d'une session de formation Galanet (Masperi et Quintin, 2007).

\begin{tabular}{|l|l|}
\hline $\begin{array}{l}\text { Phase 1 } \\
\text { Briser la glace et choix du thème }\end{array}$ & $\begin{array}{l}\text { Les participants et les équipes se présentent, font } \\
\text { connaissance et initient des interactions libres qui prennent } \\
\text { place dans les fils de discussion de l'espace Forum et dans les } \\
\text { chats. Après échanges de vue, les étudiants choisissent le } \\
\text { thème qui fera l'objet du dossier de presse. }\end{array}$ \\
\hline $\begin{array}{l}\text { Phase 2 } \\
\text { Remue-méninges }\end{array}$ & $\begin{array}{l}\text { Un premier échange libre dans le forum permet de dégager } \\
\text { les grandes pistes à débattre, qui seront à la base des futures } \\
\text { rubriques du dossier de presse. }\end{array}$ \\
\hline
\end{tabular}




\begin{tabular}{|l|l|}
\hline $\begin{array}{l}\text { Phase } 3 \\
\text { Collecte de documents et débat }\end{array}$ & $\begin{array}{l}\text { Les débats synchrones et asynchrones sont soutenus par des } \\
\text { extraits de documents déposés dans le forum. Ces éléments } \\
\text { multilingues font l'objet d'un travail de compréhension en } \\
\text { équipe encadré en présentiel par le tuteur local (enseignant } \\
\text { associé à une équipe). }\end{array}$ \\
\hline $\begin{array}{l}\text { Phase 4 } \\
\text { Réalisation et publication du dossier } \\
\text { de presse }\end{array}$ & $\begin{array}{l}\text { Le dossier de presse, structuré en rubriques, synthétise, } \\
\text { articule et illustre, par une sélection de documents écrits et } \\
\text { sonores, les passages clés des échanges entre les étudiants. }\end{array}$ \\
\hline Epilogue & Les participants font un bilan de la session et prennent congé. \\
\hline
\end{tabular}

Les phases s'organisent autour de forums de discussion. Cadrés par un scénario pédagogique et chronologique, dirigés et limités dans le temps (de deux à trois mois), avec un objectif explicite, les forums Galanet rentrent dans la catégorie des forums fermés. Il s'agit d'exposés discursifs relativement structurés aboutissant à une conclusion, alors que les forums libres sont censés se rapprocher des conversations à bâtons rompus (Sidir et Lucas, 2006).

\subsection{Le corpus}

Du point de vue de cette recherche, les forums fermés de Galanet facilitent la clôture du corpus, celle-ci étant établie par les organisateurs du dispositif, qui ferment les forums à un moment donné. D'autre part, ils garantissent un corpus plutôt homogène. En effet, ces forums sont aussi caractérisés par une unité thématique et un cadre participatif stable : le nombre des participants est établi à l'avance et leurs profils sont connus et disponibles sur la plateforme. Cela nous a permis d'isoler la variable « genre », mais aussi la variable «culture» dans un contexte plurilingue. En effet, l'appartenance ethnique, comme Ochs (1987, cité par Briggs, 1992) l'a fait remarquer, est un facteur entrant en jeu dans l'émergence de patrons que d'autres chercheurs ont mis en relation exclusivement avec le genre. C'est pourquoi nous n'avons pris en considération ici que les messages postés par des participants français (hommes et femmes). D'autre part, faisant l'hypothèse que le sentiment d'appartenance à la communauté de parole de Galanet favorise les phénomènes de reprise du discours des autres, nous avons retenu les messages des participants français les plus actifs, c'est-à-dire ayant totalisé le plus de messages. Plus spécifiquement, l'analyse a porté sur 258 messages envoyés par des hommes et 557 postés par des femmes. Il faut préciser qu'en général, dans les forums Galanet, les femmes sont plus nombreuses que les hommes. Les interactions faisant l'objet de cette étude sont issues de deux sessions de formation collaborative à distance, intitulées respectivement " L'Art du dialogue » (qui s'est déroulée du 22-01-2006 au 05-062006) et «O paleta de culturi » (qui s'est déroulée du 02-02-2009 au 22-05-2009).

\section{Analyse}

L'analyse portera de manière plus spécifique d'une part sur les phénomènes de reprise du discours du partenaire interactif (Vion, 2005); d'autre part, sur les citations et l'interdiscours (proverbes, dictons, "on dit», publicité). Les formes canoniques du discours rapporté sont rares dans notre corpus. Les modalités de reprise du discours d'autrui sont davantage à rapprocher de l'oral, de la conversation ordinaire, que de l'écrit (Devilla, 2011), comme c'est le cas dans cet échange ${ }^{3}$ :

(1) «O paleta de culturi », Phase 1, sujet de discussion «protestations »

Maryline (23/02/09 15:43)

Je pense qu'il est important de manifester son mécontentement face aux décisions gouvernementales qui nous semblent déplacées, cependant ce qui est une tradition en 
France peut-être a tort ou à raison très mal vu en Europe. De fait, en Espagne très peu de grèves sont prises au sérieux étant donné le nombre impressionnant (pour la culture espagnole) de manifestations par an. Je trouve qu'il en va de même sur le plan national. Ma conclusion est la suivante: trop de grève tue la grève....

Emunah (27/02/09 22:10)

d'accord avec Maryline, trop de grêve tue la grêve et d'autant plus qu'il me semble bon de reprendre les cours quand un nouveau texte doit être écrit: marque de respect dans les négociations et reprendre la grêve si le nouveau texte ne conviens pas. Ma position est peut-être liée à mon âge!

Laure (6/03/09 11:58)

Le droit de grève est un droit très important et un bon véhicule pour faire part de nos mécontentements aux autorités publiques, toutefois, je suis d'accord avec Maryline et Emunah, trop de grève tue le grève! Faire grève pour un oui, pour un non comme on le fait trop souvent en France revient a décrédibiliser ce genre de mouvement. Je pense qu'il faut choisir ses batailles et adapter les mouvements aux revendications - la grève n'est pas une panacée!

ElmaR (12/03/09 10:59)

En Argentina hace muchos años que protestar es un medio que utiliza el pueblo para hacerse escuchar por el gobierno; yo pienso que manifestarse a favor de una causa justa está bien, pero siempre dentro de ciertos límites de respeto y conducta. En mi país las manifestaciones son muy diversas: los maestros protestan por mejorar su salario, los productores agropecuarios por mejorar las ganancias, los universitarios por un boleto estudiantil, etc. Pero estoy de acuerdo con Maryline: "trop de grève tue la gréve".

Il y a prédominance des reformulations sur les simples répétitions en écho :

(2) «O paleta de culturi », Phase 1, sujet de discussion «quel(s) sport(s) aimezvous?»

Dominique (23/02/09 16:56)

moi, je préfère la marche à pieds, en montagne, ce qui me permet d'avoir un effort physique yout en profitant de la nature: deux en un

Laure (27/02/09 11:47)

Bonjour, Je suis assez d'accord avec Dominique; il n'y a rien de mieux qu'une bonne balade en montagne que ça soit en shorts et basquettes l'été ou combinaison de ski et raquettes l'hiver, allier sport et découverte nature est toujours très sympathique!

\subsection{Manifestation de l'accord et du désaccord}

Les locuteurs ne rapportent pas simplement une parole, ils ne se contentent pas de la reformuler, mais ils adoptent aussi vis-à-vis d'elle une attitude particulière. Ils disposent inévitablement d'un point de vue coorienté ou hétéro-orienté qui les éloignent des propos d'origine et qui justifie leur intervention. Les formes plus ou moins subtiles d'adhésion ou de distanciation permettent de faire coexister deux voix par la présence simultanée de deux énonciateurs. Dans les interactions de la plateforme Galanet les cas d'adhésion sont les plus nombreux. On peut se demander si cela dépend du contexte collaboratif et pédagogique dans lequel s'inscrivent ces échanges, basés sur un principe d'étayage réciproque et caractérisés sur le plan du contenu communicatif par une modération des propos déviants. En effet, les participants ne perdent jamais de vue qu'ils se situent dans un environnement de formation. On sait pourtant que le conflit, l' « incident critique », pour reprendre les termes de Jackson (2003, cité in Audras et Chanier, 2007), permet de déceler une réelle vie en ligne et de manifester des compétences interculturelles dans un contexte exolingue (Audras et Chanier, 2007), comme c'est le cas de Galanet. 
L'expression de l'accord ou du désaccord, comme le montrent les messages ci-dessous, est le plus souvent accompagnée de l'indication du destinataire :

(3) «O paleta de culturi », Phase 1, sujet de discussion «Quel est votre film préféré »

AlessandraA (2/03/09 11:34)

ciao mi chiamo Alessandra...anche io penso che moulin Rouge sia molto bello, è uno dei miei film preferiti...le musiche sono stupende ma la trama è un pò triste!!!!!

fafa $(6 / 03 / 0912: 15)$

Ciao a tutti !!!

J'aime beaucoup les films avec de belles grandes histoires d'amour mais avec une fin tragique, je parle en particulier de "Moulin rouge" où je rejoins Allessandra qui dit que les musiques sont magnifiques et ne sont pas en trop. Elles accompagnent parfaitement le film et la trame du film. Un autre film qui m'a marqué" C'est le temps d'un automne", une merveilleuse histoire d'amour qui prend fin encore...

(4) «O paleta de culturi », Phase 3, sujet de discussion "Amitiés entre peuples et cultures / Amitiés au-delà du temps et de l'espace »

VéroBor (9/05/09 10:59)

Je pense comme tout le monde que l'amitié entre personnes de cultures différentes est possible, seulement, si nous parlons bien ici de personnes de cultures TRES différentes, donc pas entre personnes du monde occidental par exemple, on se heurtera vite à une incompréhension mutuelle. Je pense qu'il faut avoir une ouverture d'esprit extraordinaire pour comprendre et accepter les coutumes d'une culture qui dans la nôtre sont considérées comme des actes que la morale réprouve. A mon avis ce type d'amitié existe mais il doit être très très rare.

NinaC (9/05/09 11:47)

Oui VeroBor, tu as pointé du doigt l'intérêt que suscite cette question. Il est vrai que l'on se heurte à une plus grosse difficulté si l'on parle d'amitié entre 2 personnes de culture opposée que si l'on parle de 2 personnes du monde occidental.

Or, dans notre corpus, l'expression de l'accord avec indication de l'allocutaire est l'apanage des femmes (36 cas contre 7 seulement pour les hommes). En revanche, les exemples de désaccord (ex. 5), rares dans ce type de contexte, on l'a vu, apparaissent principalement dans les messages postés par des hommes (6 cas sur 8 au total), qui de ce point de vue semblent prendre moins de précautions que les femmes. En effet, ils n'hésitent pas à prendre position, à s'opposer au discours des autres participants. Ce résultat est d'autant plus intéressant que dans notre corpus le nombre des messages postés par des hommes est nettement inférieur (presque la moitié) par rapport à celui des messages envoyés par des femmes.

(5) «O paleta de culturi », Phase 3, sujet de discussion «La vera amicizia può finire ?»

fafa (11/05/09 09:04)

Oui, si on est des vrais amis la trahison ne s'immiscera pas dans la relation.

NicolasD (14/05/09 15:35)

Pas d'accord avec toi Fafa la trahison peut malgrès tout arriver qu'on le veuille ou non

\subsection{Argument d'autorité et interdiscours}

Dans les forums de discussion, on peut relever des citations de discours externes au forum, comme par exemple la citation d'une phrase d'un auteur célèbre (Marcoccia, 2010). Ce type de citation peut avoir différentes fonctions, essentiellement argumentatives : contribuer à la constitution de l'ethos de l'auteur du message, étayer ou développer le discours citant, lui servir d'exemple, d'argument d'autorité, représenter le contre-discours, etc. (Ibid). D’après Plantin (1996 : 88), « [i]l y a argumentation d'autorité 
quand le Proposant donne pour argument en faveur d'une affirmation le fait qu'elle ait été énoncée par un locuteur particulier autorisé, sur lequel il s'appuie ou derrière lequel il se réfugie ». Dans notre corpus, peut-être en raison du contexte pédagogique des échanges, la citation est le plus souvent accompagnée de la référence du discours-source (ex. 6 et 7). Toutefois, comme le fait remarquer Marcoccia (2010) à propos des forums libres, «l'importance du phénomène citationnel dans les forums (sans doute favorisé par l'usage du copié-collé) ne s'accompagne pas du respect des règles habituellement suivies dans les textes scientifiques ». C'est le cas aussi dans la plupart des extraits de notre corpus :

(6) «O paleta de culturi », Phase 3, sujet de discussion « Etes-vous pour ou contre le mariage?»

Marie (3/03/09 20:43)

En France, le mariage aussi des us et coutumes soit religieuses soit locales. On ne parle pas de la même manière du mariage dans le monde rural, dans le monde ouvrier (dépend des régions) et des citadins. En plus, la position sociale et l'âge entrent en jeu dans la notion du mariage. Et puis, le Pacs changent un peu les données chez ceux qui veulent officialiser sans passer à l'Eglise (quelque soit l'Eglise, catholique, orthodoxe, protestants ou autres..;) L'important c'est le coeur... il faut penser comme le petit prince de St Exuperry.

(7) «O paleta de culturi », Phase 1, sujet de discussion « La place du français vs de l'anglais dans l'enseignement dans nos pays »

Alexandre (25/03/09 01:42)

Le français n'est absolument pas menacé par l'anglais. Les jacobins agitent depuis ces dernières années le spectre de l'anglais qui, d'un point de vue linguistique, n'aura pas plus d'impact en français qu'en a eu l'italien à la renaissance (à l'époque, les mêmes jacobins ont parlé de "grande menace pour la langue françoise" et proposaient d'éradiquer des mots comme scénario, cavalier, opéra, carnaval, concert, qu'ils jugeaient beaucoup trop "italiens"...). C'est un véritable fantasme français, et aussi une pointe de jalousie parce que l'anglais a détrôné le français dans son rôle de langue de relations internationale...D'ailleurs, ça me fait penser à une très belle phrase de Léopold Sedar-Senghor: "la France est parvenue à faire passer son nationalisme pour de l'universalisme." à méditer...

Dans une étude menée sur des forums libres, Von Münchow (2004) a montré la méfiance des Français vis-à-vis de la citation d'autorité. En revanche, en contexte pédagogique, on observe exactement l'inverse. Dans nos échanges plurilingues, la plupart des messages contenant des citations d'autorité sont dus à des participants français dans le forum "briser la glace », alors que les autres communautés ethnolinguistiques en présence y ont largement recours dans les forums « collecte de documents et débat » et « dossier de presse », c'est-à-dire lorsque leur emploi est sollicité par le scénario pédagogique et par les animateurs en vue de la rédaction du dossier de presse (prévu par le « contrat didactique »), comme le montre cet exemple :

(8) «O paleta de culturi », Phase 4, dossier de presse

françoise (18/05/09 10:36)

A MartyP et Sonia : dans votre texte, développez davantage la synthèse de ce qui a été dit sur Galanet. Mettez quelques citations dans toutes les langues...

Nous n'avons pourtant pas relevé de différences de genre dans l'emploi de ce type de citations, ce qui a été en revanche le cas à propos de l'interdiscours, c'est-à-dire, selon cette notion utilisée principalement en analyse du discours, de l'ensemble des discours antérieurs et à venir avec lesquels un discours déterminé est en relation implicite ou explicite (Krieg-Planque, 2012 : 189). Dans l'exemple suivant, on trouve à la fois la référence à un « on dit », à une "phrase culte », et une allusion ironique au célèbre slogan publicitaire prononcé par l'acteur américain George Clooney pour la publicité d'une marque de café connue : 
(9) «O paleta de culturi », Phase 1, sujet de discussion « L'interprétation sociologique du café »

Thibaud (23/02/09 15:39)

"Oula, mais c'est l'heure du café ! " Quelle phrase culte, Oui mais quel heure est-il, et quel type de café ...? A mon avis la diversité culturelle nous offre aussi une multitude de caractéristiques propres au café, à son rituel, à sa culture ... nul doute qu'un brésilien ou un portugais ne boivent pas le même café, de la même manière, à la même heure, et pour la même raison ! Qu'en pensez-vous ...? What else !!!!

\section{En guise de conclusion}

Cette recherche menée sur un corpus d'échanges par forum dans un contexte pédagogique a mis en évidence la préférence des femmes à rapporter le discours de leurs interlocuteurs, en adoptant un point de vue co-orienté vis-à vis de la parole des autres, à travers l'emploi de marqueurs d'approbation discursive. Comment interpréter ce phénomène relevant de ce que Sandré (2010) appelle le « dialogisme interlocutif à visée convergente »? Cette rencontre avec la parole des autres permet, nous semble-t-il, d'assurer le travail conversationnel, en développant l'argumentation. D'autre part, c'est une forme de politesse car on s'intéresse à ce que disent les partenaires interactifs et on manifeste son accord. Cela recoupe les études mettant en relief que la politesse est un trait du langage des femmes, à partir de Lakoff en passant par Brown et Levinson pour arriver aux études les plus récentes (cf. Fracchiolla, 2011). Dans le contexte d'apprentissage collaboratif de Galanet, caractérisé par le soutien et l'entraide entre apprenants, le partage et la co-construction à distance des connaissances, cela assure également une certaine convivialité de l'échange et renforce l'esprit d'équipe. Au contraire, la préférence des hommes à se démarquer des propos de leurs interlocuteurs, à adopter un point de vue hétéro-orienté vis-à-vis de la parole des autres manifeste une tendance à affirmer leur position. Cela va dans le sens du constat de Brown qui a relevé chez les hommes moins d'attention aux problèmes de «face» (cf. Kendall \& Tannen 2001: 551). Tannen, quant à elle, a mis en évidence que plusieurs rituels conversationnels remarqués chez les hommes sont basés sur une opposition rituelle ou sur l'" agonisme» (cf. Ibid. : 553). Par ailleurs, nous avons relevé ici la préférence des hommes à rapporter un discours commun. Le recours à l'argument d'autorité contribue en effet à renforcer les propos tenus sur le sujet de discussion. Ces résultats s'inscrivent ainsi dans la lignée des travaux précédents ayant montré que «les pratiques conversationnelles masculines apparaissent plus enflammées et les féminines plus conciliantes » (Bailly, 2008 : 79).

\section{Références bibliographiques}

Audras, I., Chanier, T. (2007). Acquisition de compétences interculturelles : interactions orales et écrites en tridem en ligne. Lidil, 36, 23-42.

Baider, F. (2012). Compte rendu de Alexandre Duchêne et Claudine Moïse (éds), Langage, genre et sexualité, 2011, Québec, Éditions NotaBene. Langage et société, 139, 150-154.

Bailly, S. (2008). Les hommes, les femmes et la communication (mais que vient faire le sexe dans la langue) ? Paris : L'Harmattan.

Bell, C.M., McCarthy, P.M., McNamara, D.S. (2006). Variation in Language Use Across Gender: Biological versus Sociological Theories. http://csjarchive.cogsci.rpi.edu/proceedings/2006/docs/p1009.pdf

Briggs, Cl. (1992). "Since I am a Woman, I will Chastise my Relatives": gender, reported speech, and the (re)production of social relations in Warao ritual wailing. American Ethnologist, 19, 2, 337-361.

Coates, J. (1994). No gap, lots of overlap: turn-taking patterns in the talk of women friends. In Graddold, D. et alii (éds). Researching Language and Literacy in Social Context, Clevedon: Multilingual Matters, 177-192.

Devilla, L. (2011). Le dialogisme dans les interactions pédagogiques plurilingues en ligne. In J. Bres, A. Nowakowska, J.-M. Sarale, S. Sarrazin (éds.). Actes du colloque international « Dialogisme : langue, discours » 
(8-10 septembre 2010, Montpellier), mis en ligne le 10 juillet 2011. http://www.praxiling.fr/dialogisme-languediscours.html?lang=fr

Eckert, P. (2000). Linguistic Variation as Social Practice. Oxford : Blackwell.

Eckert, P. (1989). The whole woman : sex and gender differences in variation. Language variation and change, 1, 3, 245-267.

Eckert, P., McConnell-Ginet, S. (2003). Language and gender. Cambridge : Cambridge University Press.

Fracchiolla, B. (2011). Politeness as a strategy of attack in a gendered political debate: The Royal-Sarkozy debate. Journal of Pragmatics, 43, 2480-2488.

Greco, L. (2011). Compte rendu de Talbot Mary, Language and Gender, Genre, sexualité \& société. http://gss.revues.org/index1910.html

Herring, S. (2000). Gender differences in CMC: Findings and implications. CPSR Newsletter, 18. http://archive.cpsr.net/publications/newsletters/issues/2000/winter2000/herring.htm

Herring, S. et Paolillo, J. C. (2006). Gender and genre variation in weblogs. Journal of Sociolinguistics, 10, 4, 439459.

Hert, Ph. (1999). Quasi-oralité de l'écriture électronique et sentiment de communauté dans les débats scientifiques en ligne. Réseaux, 97, 211-259. http://www.persee.fr/web/revues/home/prescript/article/reso_07517971_1999_num $17 \_97 \quad 2171$

Holmes, J. (1995). Women Men and Politeness. London: Longman.

Holmes, J. (1989). Sex differences and apologies: One aspect of communicative competence. Applied Linguistics, 10, 2, 194-213.

Holmes, J., Schnurr, S. (2006). Doing femininity at work: More than just relational practice. Journal of Sociolinguistics, Vol. 10, 1, 31-51.

Huffaker, D. A., Calvert, S. L. (2005). Gender, identity, and language use in teenage blogs. Journal of ComputerMediated Communication, 10, 2. http://jcmc.indiana.edu/vollo/issue2/huffaker.html

Kendall, S., Tannen, D. (2001). Discourse and gender. In D. Schiffrin, D. Tannen, D. et H. E. Hamilton (éds). The Hanbook of discourse analysis. Oxford : Blackwell, 548-567.

Krieg-Planque, A. (2012). Analyser les discours institutionnels. Paris : Armand Colin.

Lucas, N. (2010). Citation interactionnelle et citation emblématique dans les forums de discussion en ligne. In Communications du IVe Ci-dit. http://revel.unice.fr/symposia/cidit/index.html?id=526.

Mangenot, F. (2007). Analyser les interactions pédagogiques en ligne, pourquoi, comment? In Gerbault, J. (éds). La langue du cyberespace : de la diversité aux normes. Paris : L'Harmattan, 105-120.

Marcoccia, M. (2010). Formes et fonctions de la citation et du copier-coller dans les discussions en ligne. In Communications du IVe Ci-dit. http://revel.unice.fr/symposia/cidit/index.html?id=550.

Marcoccia, M. (2004). L'analyse conversationnelle des forums de discussion : questionnements méthodologiques. Les Carnets du CEDISCOR, 8, 23-37.

Masperi, M., Quintin, J.-J. (2007). Modèle de scénario pédagogique pour la pratique de la compréhension croisée plurilingue à distance : élaboration, usage et effets. In Actes du colloque Scénario 2007. Montréal (Québec) : Centre de Recherche 113-120. http://www.groupes.polymtl.ca/cirta/Documents/Scenarisation2007 Actes.pdf

Milroy, L. (1980). Language and social network. Oxford : Blackwell.

Mondada, L. (1999). Formes de séquentialité dans les courriels et les forums de discussion. Une approche conversationnelle de l'interaction sur Internet. Apprentissage des Langues et Systèmes d'Information et de Communication (Alsic), Vol. 2, 1, 3-25. http://alsic.u-strasbg.fr/Num3/mondada/alsic n03-rec1.htm

Ochs, E. (1992). Indexing gender. In Duranti, A., Goodwin, C. (éds). Rethinking context : Language as an interactive phenomenon. Cambridge: Cambridge University Press, 335-358. 
Ochs, E. (1987). The impact of stratification and socialization on men's and women's speech in Western Samoa. In S. Philips, C. Tanz, et S. Steele (éds.). Gender and Language. Cambridge : Cambridge University Press, 50-70.

Plantin, C. (1996). L'argumentation. Paris : Seuil.

Sandré, M. (2010). Dialogisme, comportement et débat politique télévise: Ségolène Royal lors du débat de l'entredeux tour. In Communications $d u$ IV Ci-dit. http://revel.unice.fr/symposia/cidit/?id=614

Savicki,V., Lingenfelter, D., Kelley, M. (1996). Gender language style and group composition in Internet discussion groups. Journal of Computer Mediated Communication. http://jcmc.mscc.huji.ac.i1/vol2/issue3/savicki.html

Sidir, M., Lucas, N. (2006). De l'analyse des discours à l'analyse structurale des réseaux sociaux : une étude diachronique d'un forum éducatif. Sticef, 13. http://sticef.univ-lemans.fr/num/vol2006/sidir15/sticef 2006 sidir $15 . \mathrm{htm}$

Sznycer, K. (2010). Strategies of powerful self-presentations in the discourse of female tennis players. Discourse and Society, 21, 4, 458-479.

Tannen, D. (1995). Gender and Discourse. Oxford : Oxford University Press.

Vion, R. (2005). Modalités, modalisations, interaction et dialogisme. In Bres, J. et alii (éds). Dialogisme et polyphonie. Approches linguistiques. Actes du colloque de Cerisy. Bruxelles : De Boeck Duculot, 143-156.

Von Münchow, P. (2004). Le discours rapporté dans un forum de discussion sur l'internet. Les Carnets du CEDISCOR, 8, 91-112.

Von Münchow, P. et Rakotoelina, F. (2006). L'interrogation et le discours rapporté dans les forums de discussion sur l'environnement en français et en anglo-américain. Les Carnets du CEDISCOR, 9, 93-112.

\footnotetext{
${ }^{1}$ Voir le récent appel (2013) lancé par la revue Langage et société pour un numéro spécial intitulé « Les recherches linguistiques sur le genre: bilan et perspectives ».

2 www.galanet.eu. Ce projet a été financé par le programme Socrates-Lingua2 (2001-2004) et a réuni sept partenaires : Université Stendhal-Grenoble3, Université Lumière Lyon 2, Universidade de Aveiro, Universitat Autònoma de Barcelona, Universidad Complutense de Madrid, Università degli Studi di Cassino, Université Mons-Hainaut. L'objectif de ce projet est de développer une formation à partir d'une plateforme Internet pour pratiquer et développer la compréhension croisée (ou intercompréhension) entre locuteurs de langues romanes : portugais, italien, français, espagnol, catalan et roumain. Une session de formation Galanet se déroule sur une période de plusieurs mois (deux à quatre mois en ce qui concerne les premières sessions) et peut accueillir des groupes d'étudiants relativement nombreux (plus d'une centaine d'étudiants), réunis en équipes et encadrés par des tuteurs (en moyenne un tuteur pour six étudiants). Chaque équipe est localisée en principe dans une université ou centre de formation d'un pays romanophone (Espagne, Portugal, Italie, France, Argentine, Mexique, Brésil, Roumanie). Elle dispose d'outils facilitant la collaboration à distance (outil de prise de décision collective à propos du thème à traiter, outil de réalisation collaborative de la publication finale) et l'interaction (clavardage, forum, courriel, messagerie instantanée).

${ }^{3}$ Dorénavant nous allons renvoyer au corpus de la manière suivante : session, phase, sujet de discussion, pseudonyme de l'auteur, rendu anonyme, date et heure de l'envoi du message. Les échanges du corpus ont été rapportés tels quels, sans aucune correction. Nous soulignerons systématiquement les parties des messages ciblées par le commentaire.
} 